\title{
Scanning temporally structured lists: Evidence for dual retrieval processes*
}

\author{
DAVID BURROWS \\ State University of New York, Brockport, New York 14420 \\ and \\ RONALD OKADA \\ Atkinson College, York University, Downsview, Ontario, Canada
}

\begin{abstract}
Ss were presented with lists of memory items containing one temporal pause. After each list, Ss were asked to classify a test item as positive or negative based on the memory information. In Experiments I and II, the test item was to be classified as positive if it occurred in the memory set at all, and mean reaction times for the decision were longer than for a no-pause control. In Experiment III, a positive response was required provided that the test item was in a relevant portion of the list as defined by a postlist cue. Reaction times were longer and showed a somewhat different pattern than for a no-pause control. An interpretation was offered based on the hypothetical existence of two simultaneously executed serial scans, one "exhaustive" and the other "self-terminating," as defined by S. Sternberg.
\end{abstract}

When a person needs to make use of a specific piece of memorized information, such as his home address, the resulting retrieval process is normally quite rapid and accurate. The retrieval of such information is often so rapid that it seems to be automatic and immediate. It is surprising, therefore, that most studies of simple retrieval situations suggest that retrieval is accomplished by search techniques that would be extremely inefficient whenever "target" information is embedded in large amounts of other information. Sternberg $(1966,1969)$ has produced compelling evidence that retrieval of target information, at least from short-term memory, involves an item by item exhaustive search of the available information. The evidence was obtained from a widely used paradigm in which $S s$ are required to decide whether or not a test item belongs to a short, previously memorized list of items. A positive response is required if the test item was in the memory list, and a negative response is required otherwise. The latency or reaction time (RT) for this two-choice decision is usually a linear function of the number of items in the memory list, with equal slopes for positive and negative responses. The linearity suggests serial search: The test item is compared with each memory item and, when list length is increased, the number of such comparisons increases and hence the linear increase in RT. The equal slopes suggest an exhaustive search: If the search terminated after a match was encountered, positive responses could be initiated after searching (on the average) one half of

*This research was supported in part by grants to the Center for Research in Human Learning at the University of Minnesota from the National Science Foundation (GB-17590), the National Institute of Child Health and Human Development (HD-01136 and $\mathrm{HD}-00098$ ), and the Graduate School of the University of Minnesota, and by National Rescarch Council of Canada Grant A8266 to the second au thor.

tRequests for reprints should be sent to David Burrows, Department of Psychology, State University of New York College at Brockport, Brockport, New York 14420. the memory list, and the slope for the positive function would be half as steep as the slope for the negative function.

This model seems implausible when large amounts of information are involved; some modification of the process must be necessary for any kind of efficient information processing. One possible mechanism for this modification is that whenever lists contain many items, $S$ structures or subdivides the total memory list and restricts his scan to a selected portion of the total list. If the structuring is done efficiently, many items could be eliminated without being scanned at all.

The present series of experiments introduced temporal structure into memory lists in an attempt to determine whether people can use structure to make their retrieval strategies more efficient. In each experiment, temporal pauses were introduced during memory list presentation, dividing the memory list into distinct groupings. Such temporal groupings are known to affect the retention of short memory lists (Bower, 1970; Neisser, 1967, Chapter 9) and can facilitate rapid responses when $S$ s are asked to recall an item that occurred after a test item in a memory list (DeRosa \& Baumgarte, 1971). Temporal information appears to be preserved for short periods of time after presentation. The explicit emphasis on the use of this information could influence Ss to use it in their information retrieval.

\section{EXPERIMENT I}

In Experiment I four-item memory sets were used, with a temporal pause at each possible position. The purpose of the experiment was to see whether Ss make use of the different sized sublists that result from such a procedure. For example, if a pause occurs after Item 1, this list will be separated into a one-item and a 
Table 1

Mean RT in Milliseconds as a Function of Pause Position for Positive, Negative, and Combined Trials

\begin{tabular}{lcccc}
\hline & \multicolumn{4}{c}{ Pause Position } \\
\cline { 2 - 5 } Trial Type & 1 & 2 & 3 & 4 \\
\hline Positive & 645 & 594 & 632 & 601 \\
Negative & 655 & 617 & 650 & 609 \\
Combined & 650 & 607 & 641 & 605 \\
\hline
\end{tabular}

three-item sublist. If the first item was tested for, the scan might stop at the temporal boundary and a response might be initiated after checking only the first item. This would not be true if the first item were tested for after a memory presentation with a break after the second or third item. At the very least, if Ss can make use of pauses, they should be faster at responding than if no pause at all is introduced.

\section{Method}

Subjects. The Ss were 17 undergraduates, both men and women, enrolled in the introductory psychology course at the University of Minnesota. Participation in the experiment was rewarded by adding two percentage points to the students' final course grade.

Apparatus. All stimulus materials were recorded in a male voice and played on a Wollensak tape recorder via Koss Pro-4 headphones. The materials were recorded on a single track of the tape and presented binaurally. RTs were measured to the nearest $.01 \mathrm{sec}$ by a Standard electric timer. The timer was activated by a Uher diapilot, Model F422. The diapilot operated by reading prerecorded signals from the tape that were synchronized with the presentation of test words. The $S$ signaled his response and stopped the timer by pressing one of two pushbuttons. Two small lamps indicated to $E$ which button had been pressed on each trial. A plywood partition separated $\mathrm{E}$ and $\mathrm{S}$.

Procedure. Each trial consisted of the presentation of the memory list of four single-digit numbers followed by a test digit. Presentation of memory digits was serial, at a rate of three digits every $2 \mathrm{sec}$. After presentation of the memory set, there was a 1 -sec pause followed by presentation of the test digit. $S$ was instructed to decide as quickly as possible whether or not the test digit was included in the memory set and then press one of two buttons; a positive response button if the test digit was included in the memory set and a negative response button otherwise. After a 5-sec pause, another trial began. During memory set presentation, there was a pause equal in length to the time for presenting one memory item. The pause occurred equally often after the first, second, third, or fourth memory item had been presented. The Ss were instructed to use this pause to organize the list or engage in any other activity that might make the task easier. The condition with a pause after the fourth item was considered a control. There was no pause in the memory set itself, but the total presentation time was equivalent to that for the three pause groups. Each $S$ participated in three blocks of trials. Each block consisted of four practice trials and 32 test trjals. There were eight trials for each pause position, four followed by positive test items and four followed by negative test items. On positive trials, the to-be-tested item was presented equally often in each serial position. Three different tapes were made, each consisting of four practice and 32 test trials. On each trial the digits presented were selected from a random permutation of the set $0-9$. Zero was pronounced as "Oh." Each S was presented with three tapes. The design was thus a 17 by 4 by 2 by 12 factorial, with the factors being Ss, pause position, response type (positive vs negative), and replications.

\section{Results}

Before the RT data were analyzed, all trials on which $S$ made the incorrect response were discarded. The overall error rate was $8 \%$, which is somewhat high for this type of task but low enough to justify this procedure, particularly since $30 \%$ of all errors were contributed by one $\mathrm{S}$. The error rate was higher for positive than for negative trials, $12 \%$ to $3 \%[\mathrm{t}(32)=$ $2.55, \mathrm{p}<.01]$, but was fairly uniform for the four pause positions.

Table 1 shows mean RTs for each of the eight pause position by response type (positive vs negative) combinations and combined positive and negative RTs for the four pause positions. Inspection of the table shows no positive effect of having a break in the memory set as opposed to no break (pause after the fourth item). In fact, there is a negative effect: The mean RTs for the three pause conditions combined is $28 \mathrm{msec}$ longer than the mean RT for the no-pause condition ( 633 to $605 \mathrm{msec}$ ). This negative effect is significant $[\mathrm{t}(1507)=2.49, \mathrm{p}<.01]$. The lack of positive effect for temporally segmented sets is further emphasized by examining RT as a function of grouping size. Table 2 shows mean RT on positive trials as a function of the size of the group in which the tested item was presented. Group size for a positive test item is defined as the number of items on the side of the pause boundary in which the test item was located. Separate entries are shown for cases where the test item occurred before and after the pause boundary. Data from the control condition are included for comparison purposes. If Ss were using the pause to break off scanning and report a match, RT should be correlated with group size, either for groups occurring before the pause or those occurring after the pause. Inspection of the table indicates no such effect.

\section{Discussion}

The data show no positive effects of temporal breaks in the memory set. Pauses do have a negative effect when compared with a control condition. Ss appear to have treated the memory set as an unsegmented unit, ignoring the pauses in organizing the retrieval process. The evidence suggests that the memory set is stored in a representation that closely approximates the physical stimuli. The $28 \mathrm{msec}$ extra time required to scan a list with a pause in it is not too different from the time to scan one item in this sort of task (Sternberg, 1966). If the memory list were stored as a "tape recording" with

Table 2

Mean RT in Milliseconds as a Function of Effective Group Size for Positive Items

\begin{tabular}{|c|c|c|c|c|c|c|c|}
\hline \multicolumn{4}{|c|}{$\begin{array}{c}\text { Items Occurring Before Pause } \\
\text { Effective Group Size }\end{array}$} & \multicolumn{4}{|c|}{$\begin{array}{c}\text { Items Occurring After Pause } \\
\text { Effective Group Size }\end{array}$} \\
\hline 1 & 2 & 3 & 4 & 1 & 2 & 3 & 4 \\
\hline 631 & 603 & 643 & 601 & 599 & 586 & 648 & 601 \\
\hline
\end{tabular}


one blank unit in the place of the pause trials, "replaying" or scanning such a memory list would require one extra unit of time. This interpretation suggests that the phenomenon of exhaustive scanning may be tied to the existence of short-term memory representations that are close approximations to the original stimuli. In order to scan a representation of the positive set in a self-terminating fashion, it is necessary to segment that representation and such segmentation may result in a net loss of time. Playing back an entire tape recording is easier than cutting out unrecorded sections and playing only the recorded segments.

\section{EXPERIMENT II}

Experiment I left open two questions which led to Experiment II: (1) The failure to use temporal organizations may have resulted from uncertainty about the pause locations. Not knowing where a pause will occur may disrupt encoding of the memory list and cause a slowdown that masks any positive retrieval effect of pauses. (2) The assertion that the slowdown in the pause conditions is equal to the time for scanning one item was not testable, since set size was not varied and a scan rate could not be obtained.

Experiment II attempted to answer these questions by fixing the position of the pause, so that $S$ always knew where it would come, and by varying set size for both a pause group and a no-pause control, so that comparisons between the magnitude of the negative pause effect (if any) and the scan rate could be made.

\footnotetext{
Method

Subjects. The Ss were 32 undergraduates from the same pool as in Experiment $I$.

Apparatus. The apparatus was the same as in Experiment I.

Procedure. As in Experiment I, Ss were presented with a memory set followed by a test item and were required to decide whether the test item was present in the memory set. The memory lists consisted of two, four, six, or eight items. In the experimental condition, a pause equal in length to one item was introduced after half of the memory list items had been presented. There were no pauses in memory list presentation for the control group. Items were presented at a rate of three items every $2 \mathrm{sec}$, with a 1 -sec pause between memory list and test item. Items were sampled without replacement from a pool of 620 single-syllable words with a frequency of AA or A on the Thorndike-Lorge (1944) word count. Each S served in either the control or experimental condition and was presented with four blocks of four practice and 24 test trials each. Within each block, memory list length was constant at one of the four values used. The 24 trials consisted of 12 positive and 12 negative trials. Each serial position was tested equally often on positive trials, except for List Length 8 , where for any single $S$ some positions were tested more than others, but the number of tests at each position was balanced across Ss. The order in which the four blocks were presented was randomized. Two complete sets of materials were generated and recorded, that is, two tapes for each block and experimental-control combination. For each set the lists were identical for experimental and control, except for the pauses in the experimental condition. The design was a 16 by 2 by 4 by 2 by 12 factorial, with factors of Ss, condition (experimental vs control), memory list length, response type (positive vs negative), and replications.
}

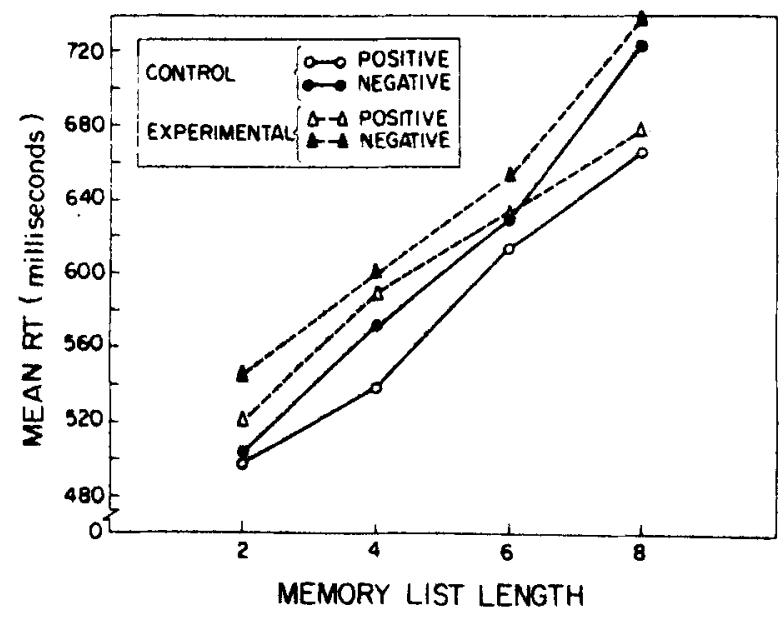

Fig. 1. Mean RT as a function of memory list length in Experiment II. Experimental vs control. Positives and negatives are shown separately.

\section{Results}

RTs for correct and incorrect responses were analyzed separately. Again the error rates were low enough to justify this procedure. For the control group the error rate ranged from less than $2 \%$ for List Length 2 to $9 \%$ for List Length 8 , with a mean of $4.6 \%$. For the experimental group the mean error rate was $6 \%$, ranging from $3 \%$ for List Length 2 to $8 \%$ for List Length 8 . Errors generally increased with set size.

Figure 1 shows mean correct RT for experimental and control groups as a function of list length. Positive and negative responses are shown separately. For each of the eight possible points of comparison, the control group had shorter RTs than the experimental group. The mean difference in RT was $28 \mathrm{msec}$. This was a significant difference $[\mathrm{t}(2116)=3.55, \mathrm{p}<.001]$. The mean RTs appear to be increasing linearly with set size in accordance with the scanning model. Linear functions fitted to the data points showed no positive-negative slope differences for control $[F(1,4)=3.91$, n.s. $]$ or experimental $[F(1,4)=3.26$, n.s. $]$. This is in accordance with the hypothesis that the scan is an exhaustive scan for both groups.

Figure 2 shows mean RT for the experimental and control groups with positive and negative responses combined. The lines shown were fit by least-squares linear regression. Both functions yield good linear fits. The slope difference between experimental and control of $3 \mathrm{msec} /$ item was not significant $[\mathrm{F}(1,4)=3.28$, n.s. $]$. The difference between the fitted intercepts was significant $[\mathrm{t}(4)=8.33, \mathrm{p}<.01]$. This pattern of results shows that memory list length affects the two groups similarly, with a constant difference reflected by the intercept effect.

\section{Discussion}

The results confirm the conclusions of Experiment $I$. A pause in the memory list actually slows down the 


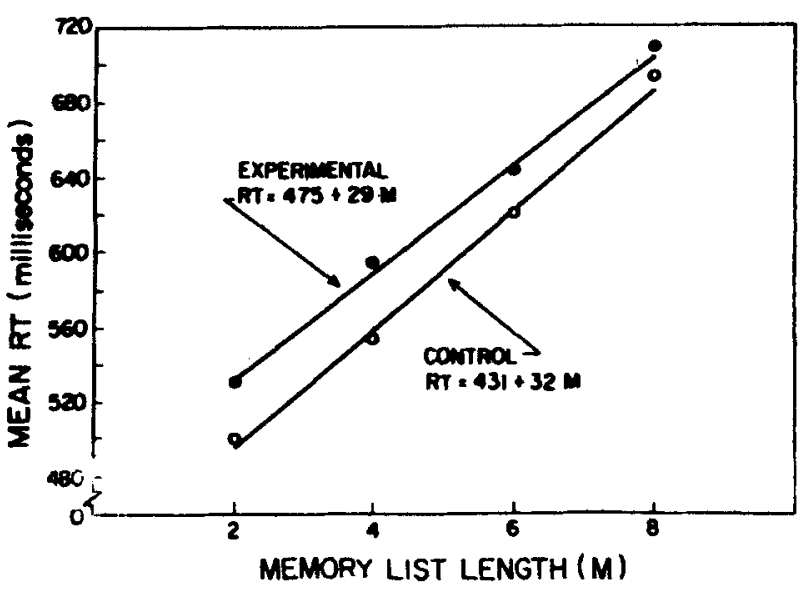

Fig. 2. Mean RT as a function of memory list length in Experiment II for combined experimental vs combined control. Straight lines fitted by linear regression.

retrieval process, even when the position of the pause is known. The actual scan rate for the experimental group is equivalent to that for the control group, as evidenced by the equal slopes for the two conditions.

This equality and the equality of slopes for experimental positive and experimental negative suggest that the experimental group scans the memory list exhaustively. The rough equivalence of the estimated scan rate (slope of the functions) and the constant additional retrieval time for the experimental group (intercept difference) supports the conclusion that the pause acts as an additional item to be scanned. Again it appears that the memory list is represented as an unsegmented record of the original stimuli that is scanned exhaustively.

The data do not imply that an exhaustive scan of an input record is always necessary. Clearly, Ss could segment the memory list and scan a "relevant" sublist if forced to do so. It could be that exhaustive scanning is most efficient under most conditions that have been studied with the Sternberg paradigm. Working with a memory set in physical form probably requires little processing, and the small delay $(28 \mathrm{msec})$ caused when there is irrelevant material on the physical record may be more than balanced out by the savings of not having to perform segmentation or other abstraction operations on the input.

\section{EXPERIMENT III}

Experiment III was a study of retrieval when Ss are forced to confine retrieval to a subportion of a temporally segmented list. This was done for two reasons: (1) To compare the efficiency of segmentation and selective scanning with the efficiency of exhaustively scanning an unsegmented list. This comparison would provide an evaluation of the claim that exhaustive scanning is in fact more efficient (at least for short list lengths). (2) To study the selective retrieval process itself. A situation where exhaustive scanning is not possible provides an opportunity to study other retrieval strategies at Ss' disposal. An understanding of nonexhaustive retrieval strategies or techniques would be important for a general retrieval model that covers complex situations (such as those involving large amounts of information) where exhaustive search seems clearly inappropriate.

In Experiment III, Ss were presented with lists segmented by a single pause. After presentation of the list, a cue was presented, indicating that one segment of the list was "relevant" and the other "irrelevant." A test item followed and a positive response was to be made only if the test item was in the relevant portion of the list. Some test items were taken from the irrelevant portion of the list. Since these required a negative response, a simple exhaustive scan of the entire list to check for a match would not be sufficient to meet the demands of the task.

\section{Method}

Subjects. The Ss were 24 undergraduates from the same pool used in Experiments I and II.

Apparatus. The apparatus was the same as that used for Experiments I and II.

Procedure. The Ss were presented with memory lists of two, four, or six items, followed by a test item to which they were to respond positively or negatively, depending upon the task instructions. For the experimental group, a temporal pause was inserted between the first and second halves of the memory list. After the entire memory list was presented, a spoken "one" or "two" was presented, indicating which half of the list was relevant. The test item followed this cue. Ss were instructed to respond positively only if the test item was included in the relevant half of the list. For each of the three list lengths, $50 \%$ of the trials had positive test items, $25 \%$ had negative items drawn from the irrelevant portion of the list (internal negatives), and $25 \%$ had negative items not presented in the memory list at all (external negatives). For the control group, there were no pauses in the presentation of the memory list and there was no cue intervening between memory list and test item presentation. Items were presented at a rate of three item s every $2 \mathrm{sec}$, with a 1.5 -sec interval between presentation of final memory item and the test item. For the experimental group, this $1.5-\mathrm{sec}$ interval was used for presentation of the relevance cue. For the control group, this interval was empty. Memory items were sampled without replacement from the pool of 620 one-syllable words used in Experiment II. Each $S$ was assigned to either the experimental or control group and was presented with a set of 21 initial practice trials followed by three blocks of trials, each block corresponding to one of the memory list lengths. Each block consisted of four practice and 24 test trials; 12 of the test trials were positive and 12 negative. For the experimental group there were six positive trials where the first half of the list was relevant and six where the second half was relevant. Six of the 12 negative trials were internal negatives and six were external negatives, divided equally among first-half relevant and second-half relevant. All possible serial positions for positives and internal negatives were tested equally often. For the control group, serial position of positive tests was also balanced. The order in which each $S$ participated in the three blocks was randomized. Two complete sets of materials were constructed and taped, with half of the Ss under each treatment combination receiving each set.

\section{Results}

RTs for correct and incorrect responses were analyzed separately. The mean error rate for the control group 
was $8 \%$, ranging from $4 \%$ for List Length 2 to $12 \%$ for List Length 6 . For the experimental group, the mean error rate was $4 \%$, ranging from a low of $1.3 \%$ for List Length 4 to a high of $8.4 \%$ for List Length 6 .

Figure 3 shows mean RT as a function of memory list length for the experimental and control groups. Data for positive and negative trials are shown separately. The fitted lines were obtained by least-squares linear regression. Analyses of the fitted slopes showed no difference between the positive and negative functions for either the experimental $[F(1,2)<1]$ or the control group $[\mathrm{F}(1,2)=1.20$, n.s. $]$. When the combined function for all experimental conditions is compared with the function for all control conditions, the slope for the experimental group is significantly steeper than the slope for the control group $[F(1,2)=47.78$, $p<.05]$. This slope difference was confirmed by an analysis of variance of each S's mean RTs for correct responses, which showed a main effect for condition (experimental vs control) $[F(1,11)=5.97, p<.05]$ and an interaction of condition (experimental vs control) with memory list length $[F(2,22)=6.09, p<.01]$. The processing involved for the experimental group is less rapid than the processing involved for the control group.

Figure 4 shows mean RT as a function of list length for the three types of experimental trials: positive, internal negative, and external negative. The fitted functions were obtained by least-squares linear regression. The functions appear to be reasonably linear, but with very different slope constants: $80 \mathrm{msec} /$ item for positive, $121 \mathrm{msec} /$ item for internal negative, and $50 \mathrm{msec} /$ item for external negative. The difference in slopes for the two negatives suggests that two different processes may determine the events leading to a correct negative response: a relatively fast process for rejecting negative items never presented before and a slower process to reject items that have been presented but are now defined as negative. The difference in slopes, $71 \mathrm{msec}$, was significant $[\mathrm{F}(1,2)=61.28, \mathrm{p}<.05]$.

The slope of $50 \mathrm{msec} /$ item for the external negatives

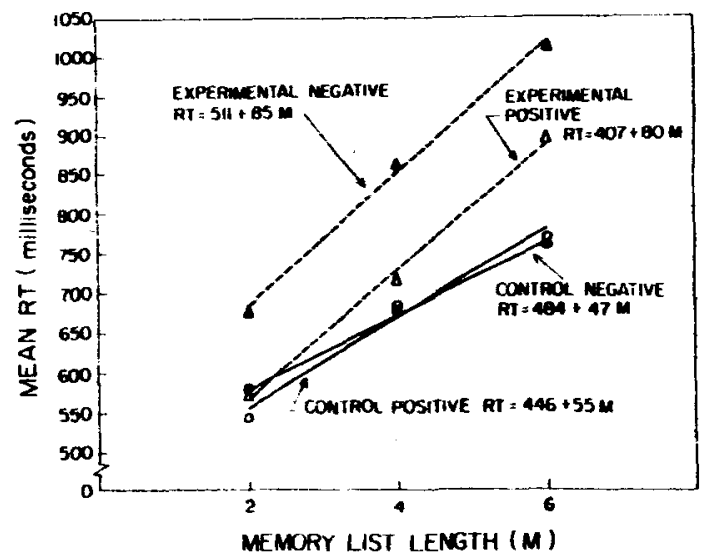

Fig. 3. Mean RT as a function of memory list length in Experiment III. Experimental vs control and positives vs negatives are separated. Straight lines fitted by linear regression.

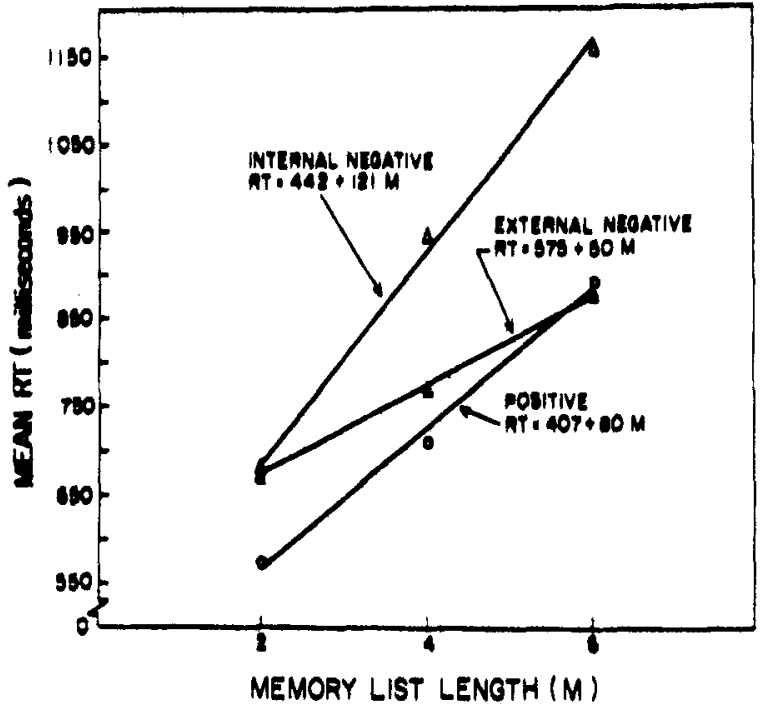

Fig. 4. Mean RT as a function of memory list length in Experiment III, experimental condition. Separate functions for positives, internal negatives, and external negatives. Straight lines fitted by linear regression.

is very close to the slope values for the control group ( $55 \mathrm{msec} /$ item for positive, $47 \mathrm{msec} /$ item for negative), suggesting that the same process is involved for control Ss and for experimental Ss on those trials where external negatives are presented.

The internal negative condition is not only considerably slower than the external negative but it is also in a $1.5: 1$ ratio with rate of scanning for the positive experimental condition, which is usually not the case in the standard Sternberg paradigm and suggests that complex processes may be occurring in the experimental condition.

\section{GENERAL DISCUSSION}

The results of Experiment III suggest two things about memory retrieval: (1) There are at least two underlying processes involved in retrieving information under the conditions of that experiment. The internal negatives/external negatives comparison demonstrates a situation where two different types of stimuli requiring the identical response result in different empirical functions. Something must be different about the processes intervening between these two types of stimuli and their common response. (2) Whatever these two processes are, they must be identical in their early stages or, if not identical, initiated simultaneously. It is not logically possible to select one of two different processes for the two categories of stimuli since the proper category for each is not known until processing has been completed.

An interpretation that would be consistent with the data from these experiments follows: When a standard Sternberg paradigm is used, Ss engage in two scanning processes which are initiated simultaneously. Each 
process is a serial scan, but they are parallel to each other in the sense that they are carried on simultaneously. Processing terminates and a response is initiated when the minimum information necessary for a response has been retrieved, usually whenever the faster of the two scans for that particular trial is finished. This general type of scanning model has been called a parallel scanning model by Burrows and Okada (1973). In the present interpretation, Scan Process 1 is a high-speed exhaustive scan of some representation of the positive set. Experiments I and II suggest that the representation used in Process 1 is a "copy" of the memory stimuli. Logically, little abstraction of the memory information is necessary; Process 1 can be thought of as a low-level scan. Scan Process 2 is a self-terminating scan of positive set items. Items are examined one by one, and a check is made after each comparison to see if a match was found. This process requires segmentation of the memory information before scanning; there must be clear boundaries between items. In Experiment III some "tagging" of memory items to indicate whether or not they are relevant would also be necessary. Process 2 can be thought of as a hypothesis testing or "high-level" scan requiring more attention and abstraction than Process 1.

In most experiments using the Sternberg paradigm, including the control condition of Experiment III, either Process 1 or Process 2 could lead to the correct response. If both are carried out, a response is initiated whenever the faster of the two for that trial is finished. In the experimental condition of Experiment III, both processes are attempted. For the external negatives, either process could yield all necessary information and a negative response results when the faster of the two processes is finished. For internal negatives and positives, exhaustive scanning is of no help. A match will always be found, but the existence of a match is not sufficient to decide on the proper response. Process 2 must be completed. Since Process 2 is slower than the faster of Processes 1 and 2, a steeper slope results for positives and internal negatives than for external negatives. The effect of set size should be equivalent for positives and internal negatives, since in each case a Process 2 scan continues until an item matching the test item is found. The slopes for positives and internal negatives should, therefore, be equivalent. The observed values are not equivalent; the internal negatives have a steeper slope than do the positives. Some appeal to a still more complicated strategy must be made. If the Ss usually start by scanning the relevant portion of the list, they would find positives after less checking than required to find internal negatives. Positives would be nearer the beginning of the scanning order and would not be as affected by set size changes. The positives would then have a less steep slope than the internal negatives. The exact ratio of the two slopes depends on what proportion of the scans actually begin with the relevant portion of the list.

The above interpretation is consistent with data and models from other scanning studies. It provides the possibility of obtaining serial position and expectancy effects in scanning, since there is a self-terminating subprocess. It is consistent with the Burrows and Okada (1973) data showing the existence of parallel scans at several levels, and it is consistent with RT data suggesting that multiple memory codes of items may exist simultaneously and be available for retrieval operations (Posner, 1969). Whatever the strengths or weaknesses of the present model, it is clear that under conditions such as those of Experiment II, the low-level type scan demonstrated in Experiments I and II is no longer sufficient for retrieval. It is equally clear that more than one retrieval process is operating in Experiment III and may be operating under other conditions as well. Any comprehensive model of retrieval must account for at least two and probably more of these underlying operations.

\section{REFERENCES}

Bower, G. H. Organizational factors in memory. Cognitive Psychology, 1970, 1, 18-46.

Burrows D., \& Okada, R. Parallel scanning of semantic and formal information. Journal of Experimental Psychology, $1973,97,254-257$.

DeRosa, D. V., \& Baumgarte, R. Probe digit recall of items from temporally organized memory sets. Journal of Experimental Psychology, 1971, 91, 154-158.

Neisser, U. Cognitive psychology. New York: Appleton-Century-Crofts, 1967.

Posner, M. I. Abstraction and the process of recognition. In $G$. $\mathrm{H}$. Bower and J. T. Spence (Eds.), The psychology of learning and motivation: Advances in research and theory. Vol. III. New York: McGraw-Hill, 1969. Pp. 152-179.

Sternberg, S. High-speed scanning in human memory. Science, $1966,153,652-654$.

Sternberg, S. Memory scanning: Mental processes revealed by reaction-time experiments. American Scientist, 1969, 57 , 421-457.

Thorndike, E. L., \& Lorge, I. The teacher's word book of 30,000 words. New York: Columbia University Press, 1944.

(Received for publication November 5, 1873; accepted November 26,1973 .) 